\title{
Spectroscopy of photonic band gaps in mesoporous one-dimensional photonic crystals based on aluminum oxide
}

\author{
V.S. Gorelik ${ }^{1, *}$, Yu.P. Voinov ${ }^{1}$, V.V. Shchavlev ${ }^{2}$, Dongxue Bi ${ }^{3}$, Guo Liang Shang ${ }^{4}$, and \\ Guang Tao Fei ${ }^{4}$ \\ ${ }^{1}$ Lebedev Physical Institute RAS, 119991 Moscow, Russia \\ ${ }^{2}$ Moscow Institute of Physics and Technology, 141700 Dolgoprudny, Russia \\ ${ }^{3}$ Bauman Moscow State Technical University, 105005 Moscow, Russia \\ ${ }^{4}$ Hefei Institutes of Physical Science, Chinese Academy of Sciences, 230031 Hefei, China
}

\begin{abstract}
Mesoporous one-dimensional photonic crystals based on aluminum oxide have been synthesized by electrochemical etching method. Reflection spectra of the obtained mesoporous samples in a wide spectral range that covers several band gaps are presented. Microscopic parameters of photonic crystals are calculated and corresponding reflection spectra for the first six band gaps are presented.
\end{abstract}

At present, photonic crystals [1] are intensively investigated. A characteristic feature of such materials is the presence of photonic band gaps (stop bands) in their spectrum. The simplest example of such structures is a one-dimensional photonic crystal composed of the layers of alternating thickness $a_{1}, a_{2}$ and refractive index $n_{1}, n_{2}$ [2]. One of the methods to manufacture such structures is the technique of electrochemical etching [3]. Mesoporous photonic crystal films based on anodic $\mathrm{Al}_{2} \mathrm{O}_{3}$ are interesting for exploration [4-6]. These structures demonstrate high melting temperature, chemical inertness, high thermal conductivity, etc. In this study, the samples of one-dimensional aluminum-oxide-based photonic crystal films were synthesized with different crystal lattice periods in the range of 350-450 nm. These samples provide photonic stop bands in a wide spectral range from the near IR to UV.
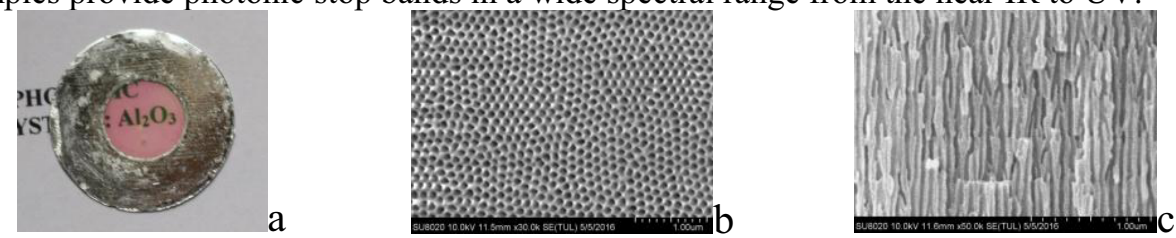

Fig. 1. (a) Surface of the photonic crystal film obtained with frontal shooting configuration; a scanning electron microscopy image of the of the film upper surface (b) and its lateral side surface (c). Scanning scale division is $0.1 \mu \mathrm{m}$.

A photograph of one of the samples obtained for frontal shooting configuration is shown at Fig. 1a. Photonic-crystal aluminum oxide film changes its color (iridescent) when we change the viewing angle. Fig. 1 (b, c) shows the microscopic structure of the front and lateral side surfaces of the film. The analysis of these surfaces provided the values of the pore

*Corresponding author: gorelik@sci.lebedev.ru 
diameter $d=55-85 \mathrm{~nm}$, the porosity $\delta=0.45$ of the upper layer of the sample (Fig. 1b) and the period $a=386 \mathrm{~nm}$ of the photonic crystal (Fig. 1c). The spectra of broadband optical radiation reflected from the surface of photonic crystal on anodic $\mathrm{Al}_{2} \mathrm{O}_{3}$ were recorded. The reflectance spectra corresponding to the first three photonic bang gaps were obtained by different manner; the results are shown in Fig. 2a.

The theory [7] was used to describe optical properties of the periodic structures and establish corresponding dispersion relation for electromagnetic waves in one-dimensional periodic structures. The dependence of reflection coefficient on wavelength was calculated with the use of the obtained dispersion relation with the dispersion of the refractive indices of the layers taken into account (Fig. 2b). Such spectrum covers first six photonic stop bands of the investigated photonic crystal.
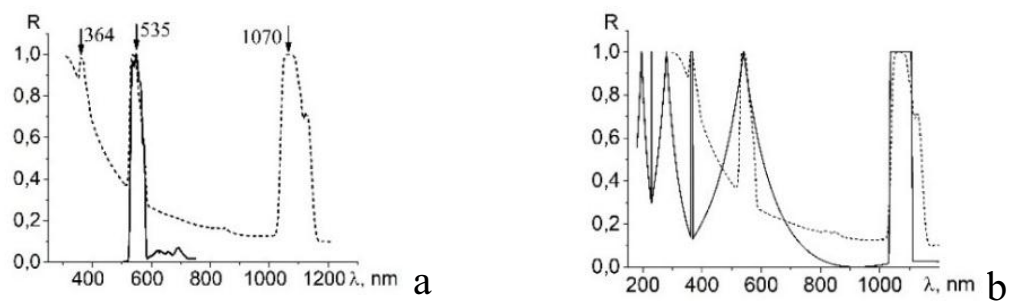

Fig. 2. (a) The reflection spectrum for a mesoporous one-dimensional $\mathrm{Al}_{2} \mathrm{O}_{3}$ photonic crystal obtained experimentally; (b) The reflection spectra obtained experimentally (dotted line) and the calculation results (solid line).

Table 1 provides a comparison between the calculation results and experimental data for the considered photonic crystal.

Table 1. Characteristics of the photonic crystal film sample obtained experimentally (exp) and theoretically (th) ( $\mathrm{m}$ - number of stop band; $\lambda_{\exp }, \lambda_{\mathrm{th}}$ - the spectral position of the center of the stop band; $\Delta \lambda_{\exp }, \Delta \lambda_{\text {th }}$ - the width of the stop band; $n_{\text {ef }}\left(\lambda_{m}\right), n_{1}\left(\lambda_{m}\right), n_{2}\left(\lambda_{m}\right)$ - the effective refractive

index and the refractive indices of the layers; $a_{\mathrm{exp}}, a_{\mathrm{th}}$ - the period of the structure)

\begin{tabular}{|c|c|c|c|c|c|c|c|c|c|}
\hline $\mathrm{m}$ & $\lambda_{\text {exp }}, \mathrm{nm}$ & $\lambda_{\text {th }}, \mathrm{nm}$ & $\Delta \lambda_{\text {exp }}, \mathrm{nm}$ & $\Delta \lambda_{\text {th }}, \mathrm{nm}$ & $n_{1}\left(\lambda_{m}\right)$ & $n_{2}\left(\lambda_{m}\right)$ & $n_{\text {ef }}\left(\lambda_{m}\right)$ & $a_{\text {exp }}, \mathrm{nm}$ & $a_{\text {th }}, \mathrm{nm}$ \\
\hline 1 & 1072 & 1070 & 78 & 78 & 1.464 & 1.305 & 1.387 & 386 & 385.8 \\
\hline 2 & 535 & 539 & 41 & 40 & 1.475 & 1.314 & 1.397 & 386 & 385.8 \\
\hline 3 & 364 & 365 & - & 24 & 1.491 & 1.344 & 1.415 & 386 & 385.8 \\
\hline 4 & - & 279 & - & 16 & 1.510 & 1.380 & 1.446 & 386 & 385.8 \\
\hline 5 & - & 228 & - & 13 & 1.541 & 1.411 & 1.477 & 386 & 385.8 \\
\hline 6 & - & 196 & - & 9 & 1.579 & 1.467 & 1.524 & 386 & 385.8 \\
\hline
\end{tabular}

Thus, the proposed scheme of determining the values of the microscopic characteristics of the mesoporous photonic crystal allows to obtain the reflection spectra and use them for determination of the spectral positions of the photonic stop bands. The synthesized photonic crystal films can be used as notch filters in the infrared and visible regions of the spectrum, as selective laser resonator mirrors and substrates for the effective sensors of molecular structures.

\section{References}

1. E. Yablonovich, Phys. Rev. Lett. 58, 2059 (1987)

2. V.S. Gorelik, Quant. Electron. 37, 409 (2007)

3. L. Pavesi, Rivista del Nuovo Cimento 20, 1 (1997)

4. L.Yisen, Y. Chang, L. Zhiyuan, L. Yi, H. Xing, Electrochem. Commun. 13, 1336 (2011)

5. V.S. Gorelik, V.V. Shchavlev, Bull. of the Lebedev Physics Institute 43, 189 (2016)

6. V.S. Gorelik et al., Opt. Spectrosc. 120, 534 (2016)

7. A. Yariv, P. Yeh, Optical waves in crystals: Propagation and control of laser radiation (Wiley, New York, 1984) 